\title{
Image Denoising and Decomposition with Total Variation Minimization and Oscillatory Functions
}

\author{
LUMINITA A. VESE* AND STANLEY J. OSHER ${ }^{\dagger}$ \\ Department of Mathematics, University of California, Los Angeles, 520 Portola Plaza, Los Angeles, \\ CA 90095-1555, USA \\ lvese@math.ucla.edu \\ sjo@math.ucla.edu
}

\begin{abstract}
In this paper, we propose a new variational model for image denoising and decomposition, witch combines the total variation minimization model of Rudin, Osher and Fatemi from image restoration, with spaces of oscillatory functions, following recent ideas introduced by Meyer. The spaces introduced here are appropriate to model oscillatory patterns of zero mean, such as noise or texture. Numerical results of image denoising, image decomposition and texture discrimination are presented, showing that the new models decompose better a given image, possible noisy, into cartoon and oscillatory pattern of zero mean, than the standard ones. The present paper develops further the models previously introduced by the authors in Vese and Osher (Modeling textures with total variation minimization and oscillating patterns in image processing, UCLA CAM Report 02-19, May 2002, to appear in Journal of Scientific Computing, 2003). Other recent and related image decomposition models are also discussed.
\end{abstract}

Keywords: functional minimization, partial differential equations, image denoising, image decomposition, texture modeling

\section{Introduction}

Recently, a new concept and a new task have been introduced in image analysis: decompose a given image $f: \Omega \rightarrow \mathbb{R}$ into two components $u+v$, such that $u$ is a cartoon representation of $f$, a simplified piecewisesmooth approximation, while $v$ is the oscillatory component, consisting of texture and noise. This new image analysis task has been first formulated in theory by Meyer [27], and the authors of the present paper have proposed a first practical new model to achieve this task in [40]. In this present paper, we develop further the model introduced in [40], discuss some of its variants, and illustrate its range of applications to image denoising, image decomposition into cartoon plus texture, as well as the capability of texture discrimination

\footnotetext{
* Supported in part by NSF ITR-0113439 and NIH P20MH65166. ${ }^{\dagger}$ Supported in part by NSF DMS-0074735, ONR N00014-97-1-0027 and NIH P20MH65166.
}

and texture segmentation. We also discuss other new related models from the literature, all inspired by [27] and [40].

The canonical, standard, previous models in image analysis start from the assumption that an image $f$ is the sum of two components, $f=u+v$, such that only $u$ contains the important main features of $f$. These models represent $u$ as a function of bounded variation (in $B V(\Omega)$ or in a subset, $S B V(\Omega)$ ), allowing for discontinuities along curves, therefore sharp edges and contours in the image $u$. The residual $v:=f-u$ is modeled in the standard models by a function in $L^{2}(\Omega)$. The main canonical examples are the total variation minimization of Rudin-Osher-Fatemi [35], Rudin-Osher [34], and the Mumford-Shah segmentation model [29]. The first model decomposes $f$ into $u+v$, such that $u \in B V(\Omega)$ and $v:=f-u \in L^{2}(\Omega)$, while the second model gives a decomposition $f=u+v$ with $u \in S B V(\Omega)$ (special functions of bounded variation), and $v:=f-u \in L^{2}(\Omega)$, as in the ROF model. These 
and other related models retain only the piecewisesmooth component $u$ from $f$, and remove away the texture + noise component $v:=f-u$. Other models having related properties are $[3,6,12,33]$, among other.

Instead, we use here a more refined model for describing the textured component $v$. It means using a norm which is truly sensitive to oscillating patterns, and our choice is motivated by some unexpected properties of the Rudin-Osher-Fatemi model [35], as it was remarked by Meyer. Also, some drawbacks of the standard models are reduced or eliminated in the new models. Also, more recent analysis favor the idea that the space $B V(\Omega)$ is not sufficient to model images with textures and small details properly, such as natural images. We refer the reader to Gousseau and Morel [19], where an experimental study of the distribution of bilevels of natural images is presented, showing that their total variation blows up to infinity with the increasing of resolution. A related work by the authors of [19] together with L. Alvarez is [2]. Also, Mumford and Gidas [28] explain that natural images are better modeled by Schwartz distributions which are not measurable functions.

More precisely, we assume that we have a given image $f \in L^{2}(\Omega)$, where $\Omega$ is an open and bounded domain on $\mathbb{R}^{2}$, with Lipschitz boundary. In order to recover $u$ from $f$, in $[34,35]$ it has been proposed to solve the following convex minimization problem:

$$
\inf _{u \in B V(\Omega)} F(u)=\lambda \int_{\Omega}|f-u|^{2} d x d y+\int_{\Omega}|\nabla u|,
$$

where $B V(\Omega)$ is the space of functions of bounded variation, $\lambda>0$ is a positive tuning parameter and $\int_{\Omega}|\nabla u|$ denotes the total variation of $u$. This minimization problem has minimizers in the space $B V(\Omega)$ (see [1, 12]; also [41] for a more general case, [4, 5] for other properties). New formulations using duality of more general cases are presented in [41], as well as in $[13,14]$ for the TV minimization.

A unique minimizer $u$ formally satisfies the EulerLagrange equation associated with the above minimization problem:

$$
u-f=\frac{1}{2 \lambda} \operatorname{div}\left(\frac{\nabla u}{|\nabla u|}\right)
$$

(for a more precise and rigorous calculation of the subdifferential of the functional, we refer the reader to $[12,41])$.
As Meyer pointed out [27], the residual $v=f-u$ in ROF can be expressed as $v=\operatorname{div} \vec{g}$, where $\vec{g}$ is a vector field given, in the sense of distributions, by

$$
\vec{g}=-\frac{1}{2 \lambda}\left(\frac{\nabla u}{|\nabla u|}\right),
$$

and satisfying $\vec{g} \in L^{\infty}(\Omega)$, because $\||\vec{g}|\|_{L^{\infty}(\Omega)}=\frac{1}{2 \lambda}<$ $\infty$. Therefore, the residual $v=f-u$ in the RudinOsher-Fatemi model is expressed, in the sense of distributions, as the divergence of a vector field $\vec{g} \in L^{\infty}(\Omega)$, i.e. as a generalized function, and this also belongs to $L^{2}(\Omega)$. It turns out that the space of generalized functions

$$
G(\Omega)=\left\{v=\operatorname{div} \vec{g}, \vec{g}=\left(g_{1}, g_{2}\right), g_{1}, g_{2} \in L^{\infty}(\Omega)\right\}
$$

is the space $W^{-1, \infty}(\Omega)$, the dual space to $W_{0}^{1,1}(\Omega)$.

On the other side, for each $u \in B V(\Omega)$, its total variation $\int_{\Omega}|\nabla u|$ is finite and can be expressed as:

$$
\begin{aligned}
& \int_{\Omega}|\nabla u| \\
& \quad=\sup _{\vec{g}}\left\{\int_{\Omega} u(\operatorname{div} \vec{g}) d x d y: \vec{g} \in C_{c}^{1}\left(\Omega ; \mathbb{R}^{2}\right),|\vec{g}| \leq 1\right\} .
\end{aligned}
$$

Meyer [27] suggests that, in order to better extract from $f=u+v$ the cartoon part $u \in B V(\Omega)$, and to leave in $v$ the noise or the textured components only, other weaker norms should be used for the residual $f-u$ in (1), instead of the $L^{2}$-norm. He proposes in [27] three spaces to model oscillatory functions, one of them being $G(\Omega)$. The space $G(\Omega)$ is a Banach space induced by the norm $\|v\|_{*}$, defined as the lower bound of all $L^{\infty}(\Omega)$ norms of the functions $|\vec{g}|$ where $\vec{g}=$ $\left(g_{1}, g_{2}\right),|\vec{g}(x, y)|=\sqrt{g_{1}(x, y)^{2}+g_{2}(x, y)^{2}}$ and where the infimum is computed over all decompositions $v=$ $\operatorname{div} \vec{g}$ of $v$.

We have the inclusions $W^{1,1}(\Omega) \subset B V(\Omega) \subset L^{2}$ $(\Omega) \subset G(\Omega)=\left(W^{1,1}(\Omega)\right)^{*}$, therefore the norm in $G(\Omega)$ is a weaker norm than the $L^{2}(\Omega)$ norm.

In [40] and in this present paper, we propose a new model for image decomposition into cartoon plus texture. To model the cartoon component $u$, we use, as in $[34,35]$, the space $B V(\Omega)$. To model the oscillatory component $v$, we use the spaces $W^{-1, p}(\Omega)$, dual to $W_{0}^{1, p^{\prime}}(\Omega)$, for $1 \leq p<\infty$, and where $\frac{1}{p}+\frac{1}{p^{\prime}}=1$. In practice, it is much easier to work with $W^{-1, p}(\Omega)$, instead of $W^{-1, \infty}(\Omega)=G(\Omega)$. Moreover, if $p \rightarrow \infty$, the spaces $W^{-1, p}(\Omega)$ approximate the space $W^{-1, \infty}(\Omega)$. 
Also, for any $1 \leq p<\infty$, these are larger spaces than $G(\Omega)$, and allow for different choices of weaker norms for the oscillatory component $v$, function of the desired result. The obtained new models can be easily solved in practice, for image denoising, image decomposition, and texture discrimination.

The outline of the paper is as follows: in the next section we give the description of the proposed new models, and discuss some of the properties. In Section 3 we discuss other related recent models for image decomposition, inspired from [27] and [40]. Then in Section 4 we present numerical results and comparisons, illustrating the motivations for the new models. We end the paper with a short concluding section.

For related results on oscillatory patterns in nonlinear problems, we refer to [30]. For related image restoration models based on the total variation minimization model in a wavelet framework, we refer the reader to [25] and [10, 26]. There is a very large literature on texture modeling and analysis in the computer vision and image analysis community, so it is impossible to mention all related references. We only mention in addition [8, 11, 20-24, 32, 36-38, 42, 43], among many other work.

\section{Description of the Proposed New Models}

For every $p \geq 1$, we consider the spaces of generalized functions

$$
G_{p}(\Omega)=\left\{v=\operatorname{div} \vec{g}, \vec{g}=\left(g_{1}, g_{2}\right), g_{1}, g_{2} \in L^{p}(\Omega)\right\},
$$

induced by the norm

$$
\|v\|_{G_{p}(\Omega)}=\inf _{v=\operatorname{div} \vec{g}, g_{1}, g_{2} \in L^{p}(\Omega)}\left\|\sqrt{g_{1}^{2}+g_{2}^{2}}\right\|_{p} .
$$

For every $p \geq 1$, the space $G_{p}(\Omega)$ can be identified with the space $W^{-1, p}(\Omega)$, the dual of the Sobolev space $W_{0}^{1, p^{\prime}}(\Omega)$, with $\frac{1}{p}+\frac{1}{p^{\prime}}=1$. In fact, the norm $\|v\|_{G_{p}(\Omega)}$ is a dual norm to the Sobolev norm $\|\nabla u\|_{p^{\prime}}$.

Then, we propose the following minimization problem:

$$
\begin{aligned}
& \inf _{u \in B V(\Omega), \vec{g} \in L^{p}(\Omega)^{2}} G_{p}(u, \vec{g}) \\
& =|u|_{B V(\Omega)}+\lambda\|f-(u+\operatorname{div} \vec{g})\|_{L^{2}(\Omega)}^{2} \\
& \quad+\mu\left\|\sqrt{g_{1}^{2}+g_{2}^{2}}\right\|_{L^{p}(\Omega)},
\end{aligned}
$$

or

$$
\begin{aligned}
& \quad \inf _{u \in B V(\Omega), g_{1}, g_{2} \in L^{p}(\Omega)} G_{p}\left(u, g_{1}, g_{2}\right) \\
& =\int_{\Omega}|\nabla u|+\lambda \int_{\Omega}\left|f-\left(u+\partial_{x} g_{1}+\partial_{y} g_{2}\right)\right|^{2} d x d y \\
& \quad+\mu\left[\int_{\Omega}\left(\sqrt{g_{1}^{2}+g_{2}^{2}}\right)^{p} d x d y\right]^{\frac{1}{p}}
\end{aligned}
$$

where $\lambda, \mu>0$ are tuning parameters, and $p \geq 1$.

The first term insures that $u \in B V(\Omega)$, the second term insures that $f-u \approx \operatorname{div} \vec{g}$, which together with the third term, insures that $v=f-u \approx \operatorname{div} \vec{g} \in G_{p}(\Omega)$. Clearly, if $\lambda \rightarrow \infty$ and $p \rightarrow \infty$, this model is formally an approximation of the model originally proposed by Meyer [27]. Indeed, if $\lambda \rightarrow \infty$ and $p \rightarrow \infty$, then in the limit $f-u=\operatorname{div} \vec{g}$ almost everywhere for those $\vec{g}$ with smallest $L^{\infty}(\Omega)$ norm. Therefore in the limit, the middle term in (5) will disappear, and the third term becomes $\|f-u\|_{*}$.

Formally minimizing the above energy with respect to $u, g_{1}, g_{2}$, yields the following Euler-Lagrange equations:

$$
\begin{array}{r}
u=f-\partial_{x} g_{1}-\partial_{y} g_{2}+\frac{1}{2 \lambda} \operatorname{div}\left(\frac{\nabla u}{|\nabla u|}\right), \\
\mu\left(\left\|\sqrt{g_{1}^{2}+g_{2}^{2}}\right\|_{p}\right)^{1-p}\left(\sqrt{g_{1}^{2}+g_{2}^{2}}\right)^{p-2} g_{1} \\
=2 \lambda\left[\frac{\partial}{\partial x}(u-f)+\partial_{x x}^{2} g_{1}+\partial_{x y}^{2} g_{2}\right], \\
\mu\left(\left\|\sqrt{g_{1}^{2}+g_{2}^{2}}\right\|_{p}\right)^{1-p}\left(\sqrt{g_{1}^{2}+g_{2}^{2}}\right)^{p-2} g_{2} \\
=2 \lambda\left[\frac{\partial}{\partial y}(u-f)+\partial_{x y}^{2} g_{1}+\partial_{y y}^{2} g_{2}\right] .
\end{array}
$$

If the exterior normal to the boundary $\partial \Omega$ is denoted by $n_{x}, n_{y}$, then the associated boundary conditions for $u, g_{1}$ and $g_{2}$ are:

$$
\begin{aligned}
& \frac{\nabla u}{|\nabla u|} \cdot\left(n_{x}, n_{y}\right)=0, \\
& \left(f-u-\partial_{x} g_{1}-\partial_{y} g_{2}\right) n_{x}=0, \\
& \left(f-u-\partial_{x} g_{1}-\partial_{y} g_{2}\right) n_{y}=0 .
\end{aligned}
$$

For practical purposes and computational speed, it is convenient to use in (5) and (6) the equivalent norms $\left\|\sqrt{g_{1}^{2}+g_{2}^{2}}\right\|_{p}^{p}$, instead of $\left\|\sqrt{g_{1}^{2}+g_{2}^{2}}\right\|_{p}$. Then, (5) and 
(6) can be substituted by

$$
\begin{aligned}
& \inf _{u \in B V(\Omega), \vec{g} \in L^{p}(\Omega)^{2}} G_{p}^{\prime}(u, \vec{g}) \\
& =|u|_{B V(\Omega)}+\lambda\|f-(u+\operatorname{div} \vec{g})\|_{L^{2}(\Omega)}^{2} \\
& \quad+\mu\left\|\sqrt{g_{1}^{2}+g_{2}^{2}}\right\|_{L^{p}(\Omega)}^{p},
\end{aligned}
$$

or

$$
\begin{aligned}
& \quad \inf _{u \in B V(\Omega), g_{1}, g_{2} \in L^{p}(\Omega)} G_{p}^{\prime}\left(u, g_{1}, g_{2}\right) \\
& =\int_{\Omega}|\nabla u|+\lambda \int_{\Omega}\left|f-\left(u+\partial_{x} g_{1}+\partial_{y} g_{2}\right)\right|^{2} d x d y \\
& \quad+\mu \int_{\Omega}\left(\sqrt{g_{1}^{2}+g_{2}^{2}}\right)^{p} d x d y .
\end{aligned}
$$

By these slight modifications, the obtained EulerLagrange equations contain only local terms, therefore using shorter computational time. These are:

$$
\begin{aligned}
& u=f-\partial_{x} g_{1}-\partial_{y} g_{2}+\frac{1}{2 \lambda} \operatorname{div}\left(\frac{\nabla u}{|\nabla u|}\right), \\
& \mu p\left(\sqrt{g_{1}^{2}+g_{2}^{2}}\right)^{p-2} g_{1} \\
& \quad=2 \lambda\left[\frac{\partial}{\partial x}(u-f)+\partial_{x x}^{2} g_{1}+\partial_{x y}^{2} g_{2}\right], \\
& \mu p\left(\sqrt{g_{1}^{2}+g_{2}^{2}}\right)^{p-2} g_{2} \\
& \quad=2 \lambda\left[\frac{\partial}{\partial y}(u-f)+\partial_{x y}^{2} g_{1}+\partial_{y y}^{2} g_{2}\right] .
\end{aligned}
$$

In our numerical calculations, we have tested the proposed new models for different values of $p$. For $1 \leq p \leq 20$, the results are similar. For $p \gg 20$, such as $p=50,100, \ldots$, then only textured details of smaller scale appear in the $v$ component. This shows that the parameter $p \geq 1$ from $W^{-1, p}(\Omega)$ is related with the size of a "cell" in a periodic pattern $v \in W^{-1, p}(\Omega)$. This remark was pointed out to the first author also by Kohn.

Remark. We would like to mention that in the above models and energies, the case $p=2$ corresponds to $v \in H^{-1}(\Omega)$, the dual of the space $H_{0}^{1}(\Omega)$. The corresponding particular case $p=2$ in (10) is therefore the following minimization problem:

$$
\begin{aligned}
& \inf _{u \in B V(\Omega), v \in H^{-1}(\Omega)} G_{2}^{\prime}(u, v) \\
& \quad=|u|_{B V(\Omega)}+\lambda\|f-(u+v)\|_{L^{2}(\Omega)}^{2}+\mu|v|_{H^{-1}(\Omega)}^{2} .
\end{aligned}
$$

In this case $p=2$, it can be shown that (see for instance [18]), for $v \in H^{-1}(\Omega)$, we have

$$
\begin{aligned}
|v|_{H^{-1}(\Omega)}^{2}= & \int_{\Omega}\left(g_{1}^{2}+g_{2}^{2}\right) d x d y=\underset{h_{1}, h_{2} \in L^{2}(\Omega), v=\operatorname{div} \vec{h}}{\inf } \\
& \times \int_{\Omega}\left(h_{1}^{2}+h_{2}^{2}\right) d x d y=\int_{\Omega}|\nabla P|^{2} d x d y
\end{aligned}
$$

for a unique $P \in H_{0}^{1}(\Omega)$, with $\vec{g}=\nabla P$, and therefore $v=\operatorname{div} \vec{g}=\triangle P$, or $v=\triangle^{-1} P$.

This specific structure for $p=2$, using the inverse Laplacian, has been exploited further by Osher, together with Solé and Vese, in a different fashion, in [31]. This is introduced next, as well as two other recent models for image decomposition, one of them inspired from (15), but in a wavelet framework [17], and the other one [7] inspired from [40] and [27].

\section{Other New Related Models for Image Decomposition}

Following the remarks by Meyer from [27] and the image decomposition model introduced by the authors in [40], other related models have been recently proposed in theory and practice, as follows.

The model by Osher et al. [31], as a continuation of the model in [40], can be seen as a modification of the particular case $p=2$ from (15). This gives an exact decomposition $f=u+v$, corresponding to $\lambda=\infty$ in (15). This is as follows [31]:

$$
\inf _{u \in B V(\Omega)} F(u)=|u|_{B V(\Omega)}+\lambda\left\|\nabla \Delta^{-1}(f-u)\right\|_{L^{2}(\Omega)}^{2} .
$$

In this case, $f-u=\triangle^{-1} P$, with $P \in H^{1}(\Omega),\left.\frac{\partial P}{\partial n}\right|_{\partial \Omega}=$ $0, \int_{\Omega} P(x, y) d x d y=0$. It is shown in [31] that the energy (16) can be minimized using a new fourth order nonlinear partial differential equation

$$
u=f-\Delta\left[\operatorname{div}\left(\frac{\nabla u}{|\nabla u|}\right)\right],
$$

with corresponding boundary conditions. The quantity $\operatorname{div}\left(\frac{\nabla u}{|\nabla u|}\right)$ denotes the curvature of level lines of $u$. This can be seen also as a smoothing equation, with stronger regularization effect than by the original Rudin-OsherFatemi model (2). As shown in [31], this model gives very good results for image denoising and decomposition into cartoon and texture. However, the model 
in [31] has a slow convergence rate, due to a too severe CFL condition imposed by the fourth order nonlinear PDE (efficient numerical methods need to be constructed for such an equation).

By the models from the present paper, the results are obtained very fast, in about 100 iterations. Another important advantage of the models proposed in this present paper and in [40] is that, explicitly computing the functions $g_{1}$ and $g_{2}$, allows to perform texture segmentation and discrimination. Also, the models (5) and (10) are more general, allowing for the choice of the dual norm $W^{-1, p}(\Omega)$, and do not consider only the case $p=2$ (the parameter $p$ plays the role as a scale for
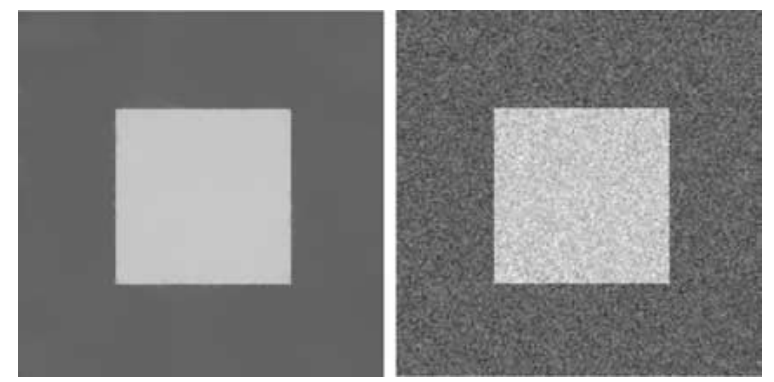

Figure 1. A synthetic original image and its noisy version.
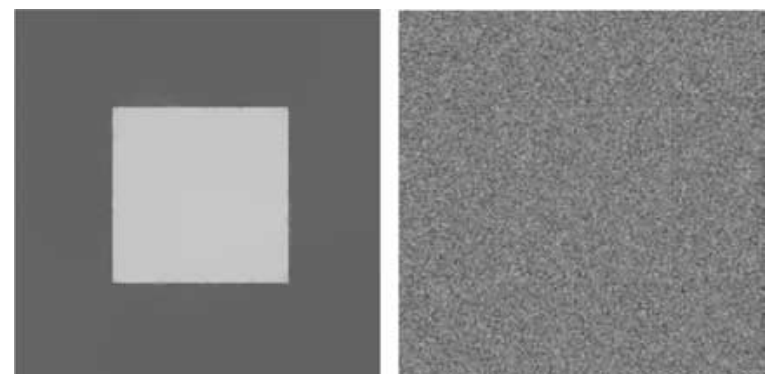

Figure 2. Minimizer $u$ and residual $f-u$ obtained using the ROF model.
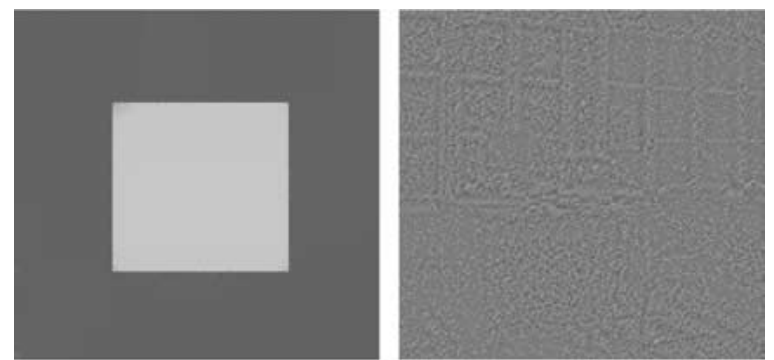

Figure 3. Minimizer $u$ and residual $f-u$ obtained using the proposed new model. the size of the cell in the periodic patterns kept in $v$ ). On the other hand, as already mentioned, an advantage of the model in [31], is that it gives an exact decomposition $f=u+v$. Comparisons of these models will be shown in the section devoted to numerical results. Finally, it is interesting to note that the model (16) gives the component $v=f-u$ as the Laplacian of the curvature of level lines of $u$, at least if $|\nabla u| \neq 0$. This shows that the textured component $v$ from (16) depends only on the level lines of the cartoon component $u$. Here, in the models from this present paper, the component $v=\operatorname{div} \vec{g}$ is much more independent of $u$.

Another model for image decomposition has been recently introduced, following [27] and [40], with a complete mathematical and theoretical formulation, by
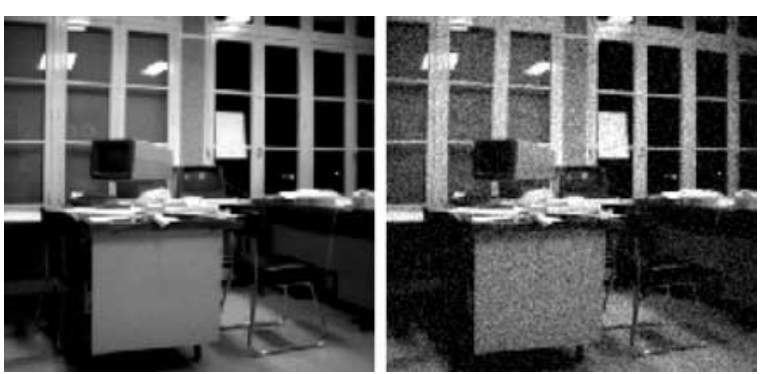

Figure 4. A real original image and its noisy version.
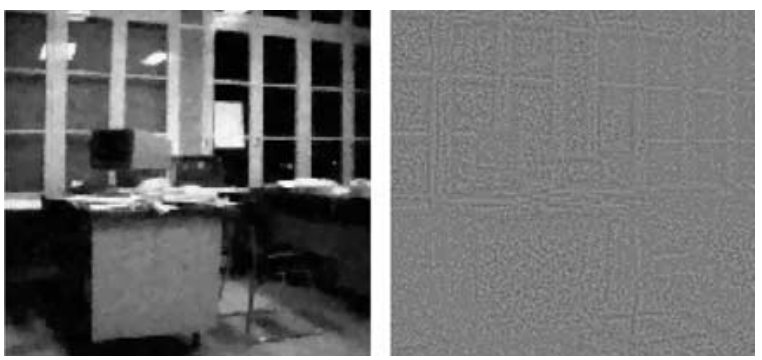

Figure 5. Minimizer $u$ and residual $f-u$ obtained using the ROF model.
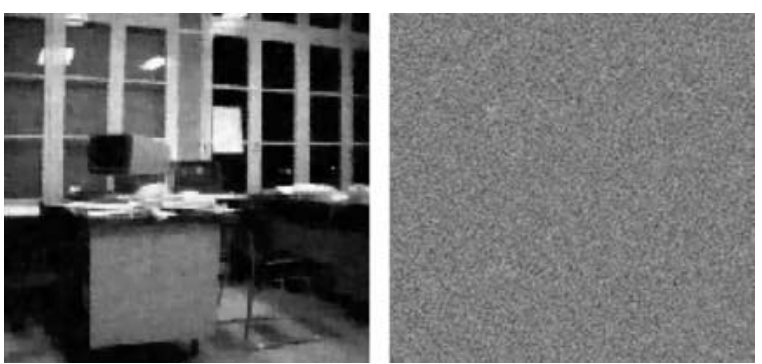

Figure 6. Minimizer $u$ and residual $f-u$ obtained using the proposed new model. 


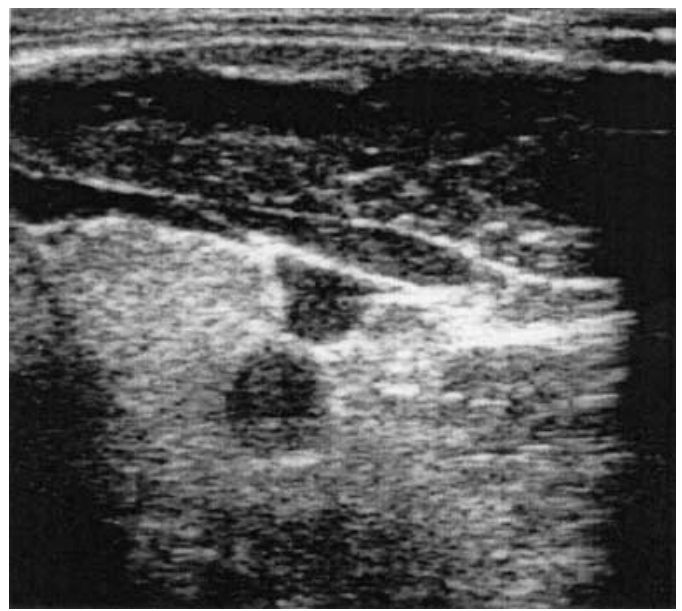

Figure 7. An initial ultrasound image $f$.

Aujol et al. [7]. The model is [7]:

$\inf _{(u, v) \in B V(\Omega) \times G_{\mu}(\Omega)}\left(\int_{\Omega}|\nabla u|+\frac{1}{2 \lambda}\|f-(u+v)\|_{L^{2}(\Omega)}^{2}\right)$,

where $G_{\mu}(\Omega)=\left\{v \in G(\Omega):\|v\|_{*} \leq \mu\right\}$, where the space $G(\Omega)$ and $\|v\|_{*}$ have been defined in the Introduction. The proposed model [7] is close to the one proposed by Meyer in [27], and it is solved using an interesting dual formulation of the total variation minimization, introduced by Chambolle in [13].

Finally, another interesting model, inspired from [40] and [31], has been recently introduced by Daubechies and Teschke [17]. The authors have reformulated our model corresponding to $p=2$ in an

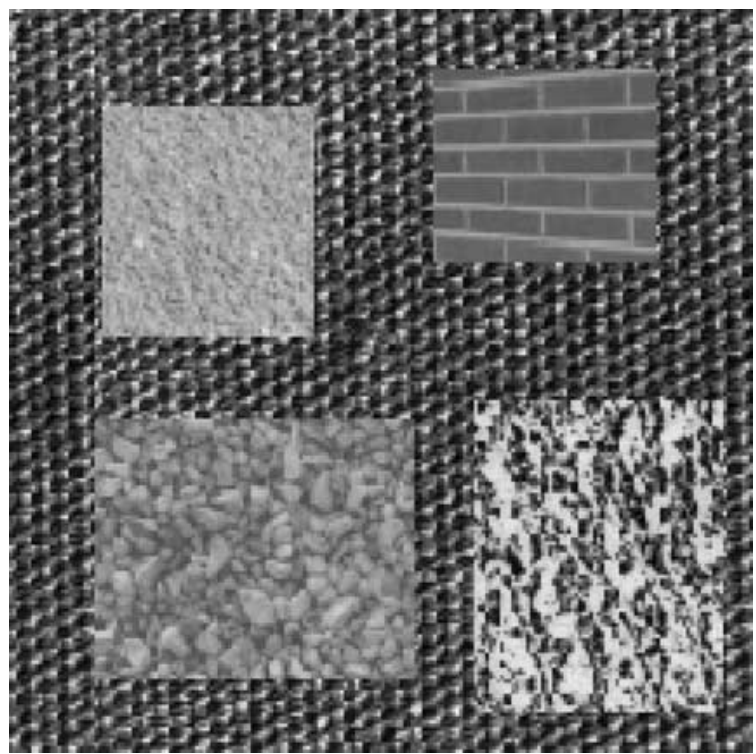

Figure 9. An initial textured image $f$.

interesting wavelet framework. This is as follows [17]:

$$
\begin{aligned}
\inf _{u, v} F(u, v)= & 2 \alpha|u|_{B_{1}^{1}\left(L^{1}(\Omega)\right)} \\
& +\|f-(u+v)\|_{L^{2}(\Omega)}^{2}+\lambda\|v\|_{H^{-1}(\Omega)}^{2},
\end{aligned}
$$

where the space $B V(\Omega)$ from (15) is replaced by the smaller Besov space $B_{1}^{1}\left(L^{1}(\Omega)\right.$ ), since this last one can be characterized in terms of wavelets coefficients. We have $B_{1}^{1}\left(L^{1}(\Omega)\right) \approx W^{1,1}(\Omega) \subset B V(\Omega)$. Very good results have been obtained using this model and more refined versions in [17]. The use of wavelets seams to be an advantage in representing well the textured details
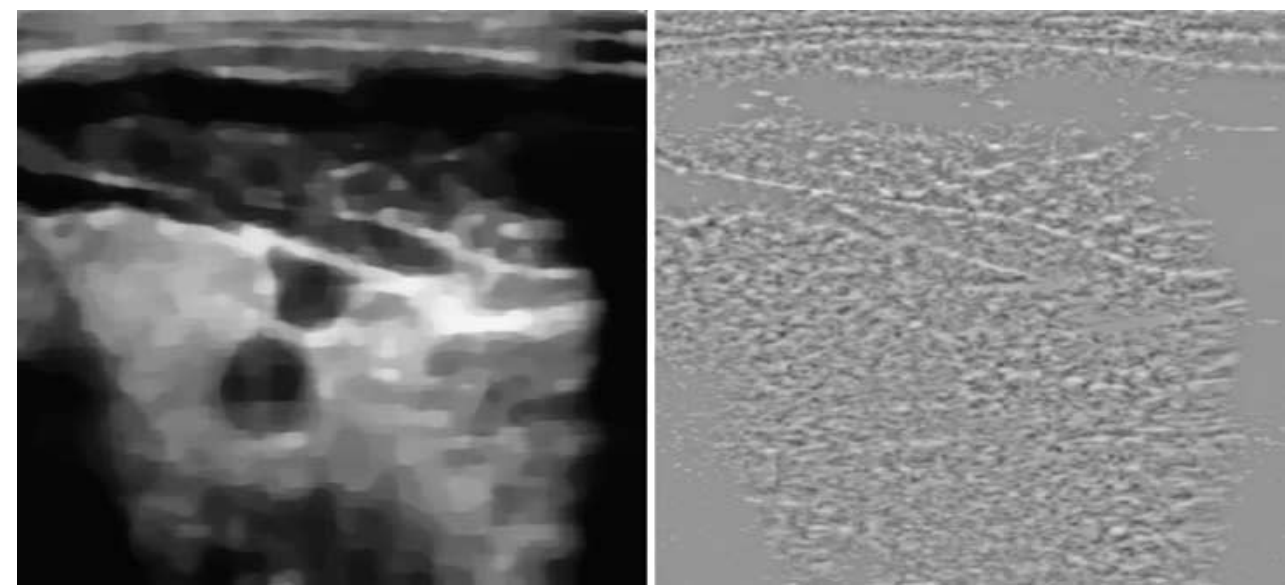

Figure 8. Results obtained with the new model: $u$ left, $v=f-u$ right, from Fig. 7. 
in the $v$ component. However, the edges are not so well preserved in the $u$ component by the space $B_{1}^{1}\left(L^{1}(\Omega)\right)$ in [17], an edge enhancement technique is also applied.

We would also like to mention that an application of the model in [40] has been introduced in [9], for structure and texture image inpainting, combined with two other existing techniques for image inpainting. Finally, following [40], very good numerical results of image decomposition into cartoon and texture have been recently obtained by Starck et al. [39] using an approach that combines the Basis Pursuit Denoising algorithm and the total variation, in a wavelet framework.

\section{Numerical Results and Comparisons}

In this section, we present numerical results of image denoising, image decomposition and texture segmentation, obtained with the proposed new models. We also show comparisons with the standard TV model of Rudin-Osher-Fatemi [35] and with the new fourth order model from [31].

In our experiments, we have used the values $p=1$ and $p=2$ in the new models. The parameter $\mu$ in the new models is kept fixed but very small, to allow highly oscillatory patterns in $v=\operatorname{div} \vec{g}$. If $\mu$ increases, than the model becomes very similar with the standard ROF model, because in that case $\||\vec{g}|\|_{L^{p}(\Omega)} \approx 0$. The parameter $\lambda$ in the new models is chosen so that the residual $f-(u+\operatorname{div} \vec{g})$ is almost identically equal to zero (in practice, this parameter ranges from 0.1 to 1). Slightly different results can be obtained for different parameters, however the dependence with respect to parameters is not too sensitive, especially in the range of parameters obtained in the experimental results.

We would also like to point out that, if the image $f$ contains both random noise and texture, then the component $v=\operatorname{div} \vec{g}$ will capture both the texture and the noise. The same situation happens in other image decomposition models into cartoon plus texture, for instance in the model [31].

In Fig. 1 we show an original synthetic image of a square, and a noisy version. In Fig. 2, we show the minimizer $u$ and the residual $f-u$ obtained with the standard TV model (the RMSE between $u$ and the original is 0.01194 , and the SNR $=27.8329$ ). The image is very well denoised. However, if we look carefully at the residual $f-u$, we can still see the shape of the square. Next, in Fig. 3, we perform the same test with the proposed new model for $p=1$. The image is also very well denoised, and the RMSE between $u$ and the original in this case is smaller, 0.00909, and the SNR = 30.4735; moreover, we do not see any "geometry" from the square object in the residual $f-u$. So the new model separates better the cartoon component from the noisy oscillatory component. For each experiment, the parameters are estimated so that the best RMSE is obtained. We use here for the RMSE and the SNR the expressions

$$
\begin{aligned}
R M S E= & \frac{\sqrt{\sum_{i=1, M, j=1, N}\left(u_{i, j}-u_{\text {orig }, i, j}\right)^{2}}}{M N}, \\
S N R= & 10 \log 10 \quad \\
& \times \frac{\sum_{i, j}\left[u_{i, j}-\frac{\sum_{i, j} u_{i, j}}{M N}\right]^{2}}{\sum_{i, j}\left[u_{i, j}-u_{\text {org }, i, j}-\frac{\sum_{i, j}\left(u_{i, j}-u_{\text {org }, i, j}\right)}{M N}\right]^{2}} .
\end{aligned}
$$

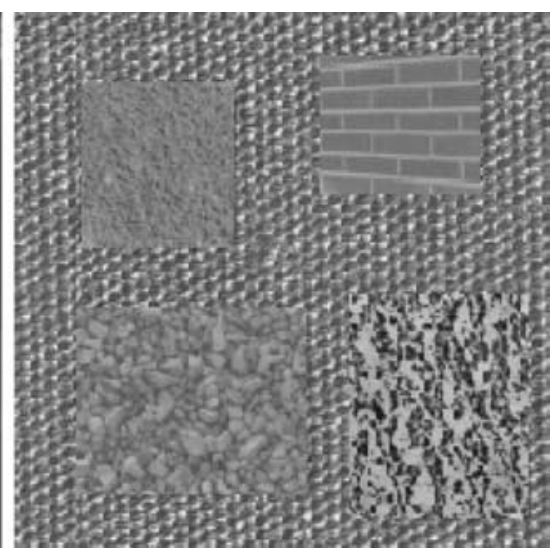

Figure 10. Image decomposition: the minimizer $u$ obtained with the proposed new model (left) and the residual $v=f-u$ (right). 


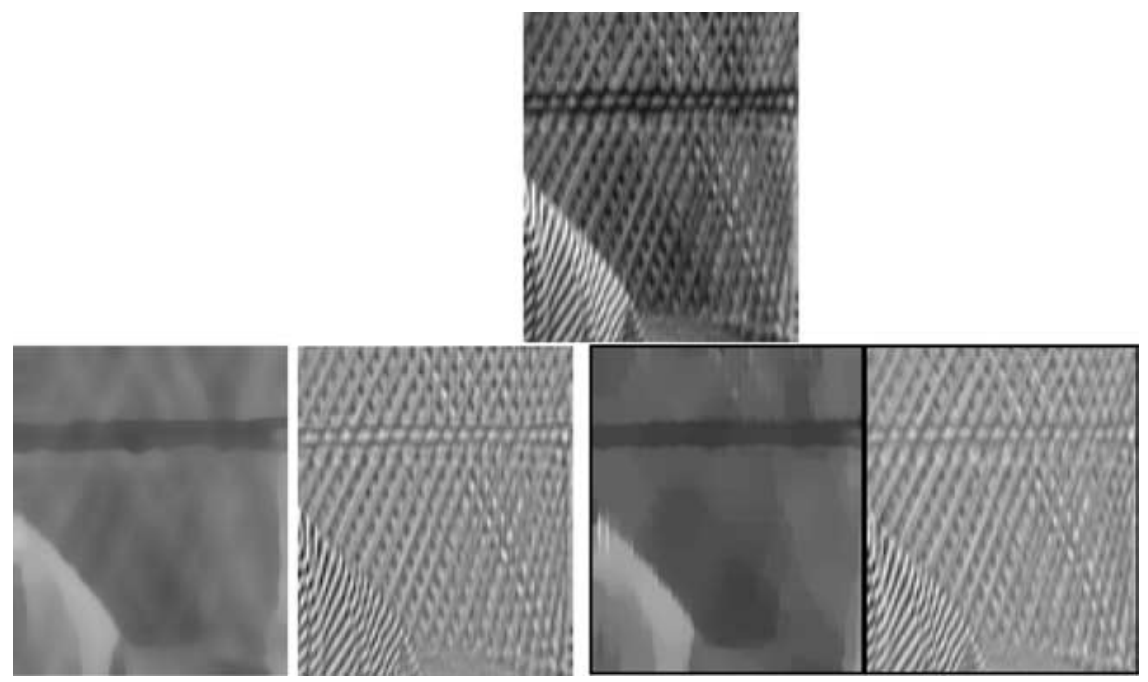

Figure 11. Comparisons of the new image decomposition models: results obtained with the model [31] (left), and with the model proposed here (right).

Initial fabric image $f$

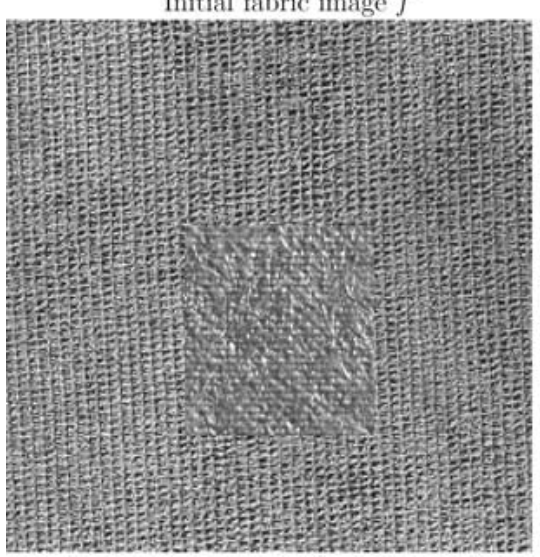

$u$

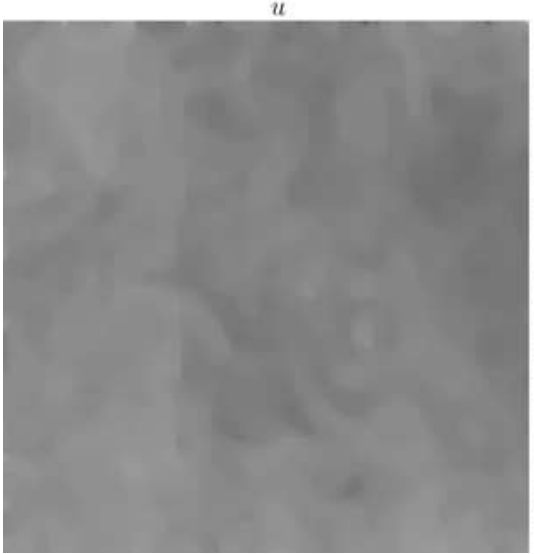

Result $u+v$

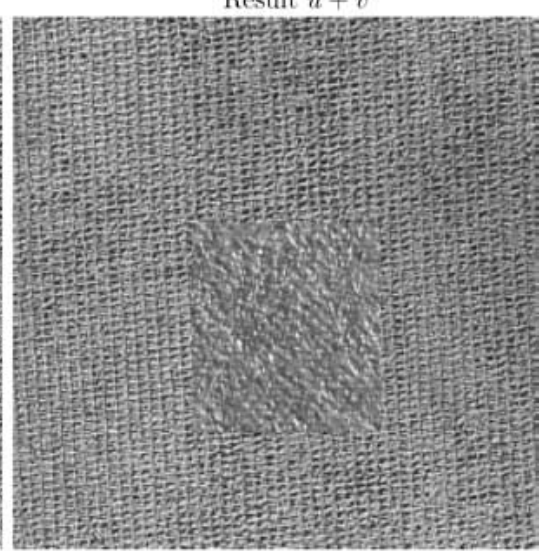

$150+0.84 v$

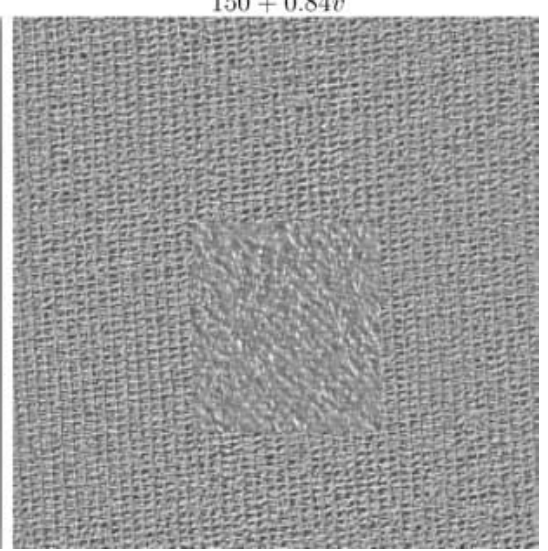

Figure 12. Image decomposition applied to a fabric textured image. 
In Figs. 4-6 we show a similar experiment, on a real image representing an office. In Fig. 4 we show the original image and a noisy version. In Fig. 5 we show the minimizer $u$ and the residual $f-u$ obtained with the standard TV model (the RMSE between $u$ and the original is 0.05809 , and the $\mathrm{SNR}=17.6823$ ). We note

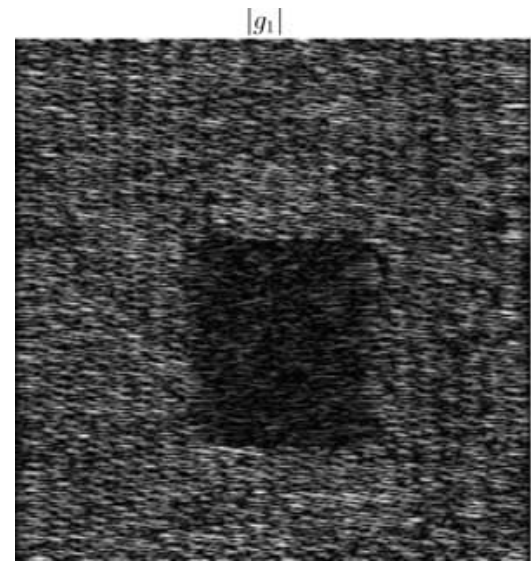

that geometry and contours of the large objects are still kept in the residual $f-u$. In Fig. 6, we perform the same test with the proposed new model. The RMSE between $u$ and the original in this case is 0.05771 , and the $\mathrm{SNR}=17.7735$. If we look at the residual $f-u$, we do not see so much "geometry" from the large features.

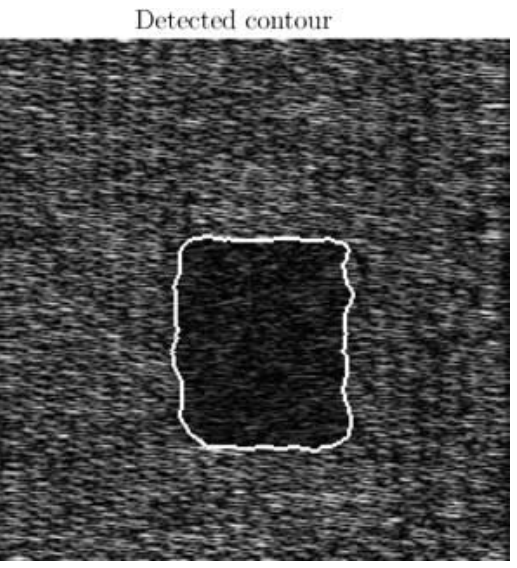

Figure 13. The component $\left|g_{1}\right|$ and detected contour for the fabric image.
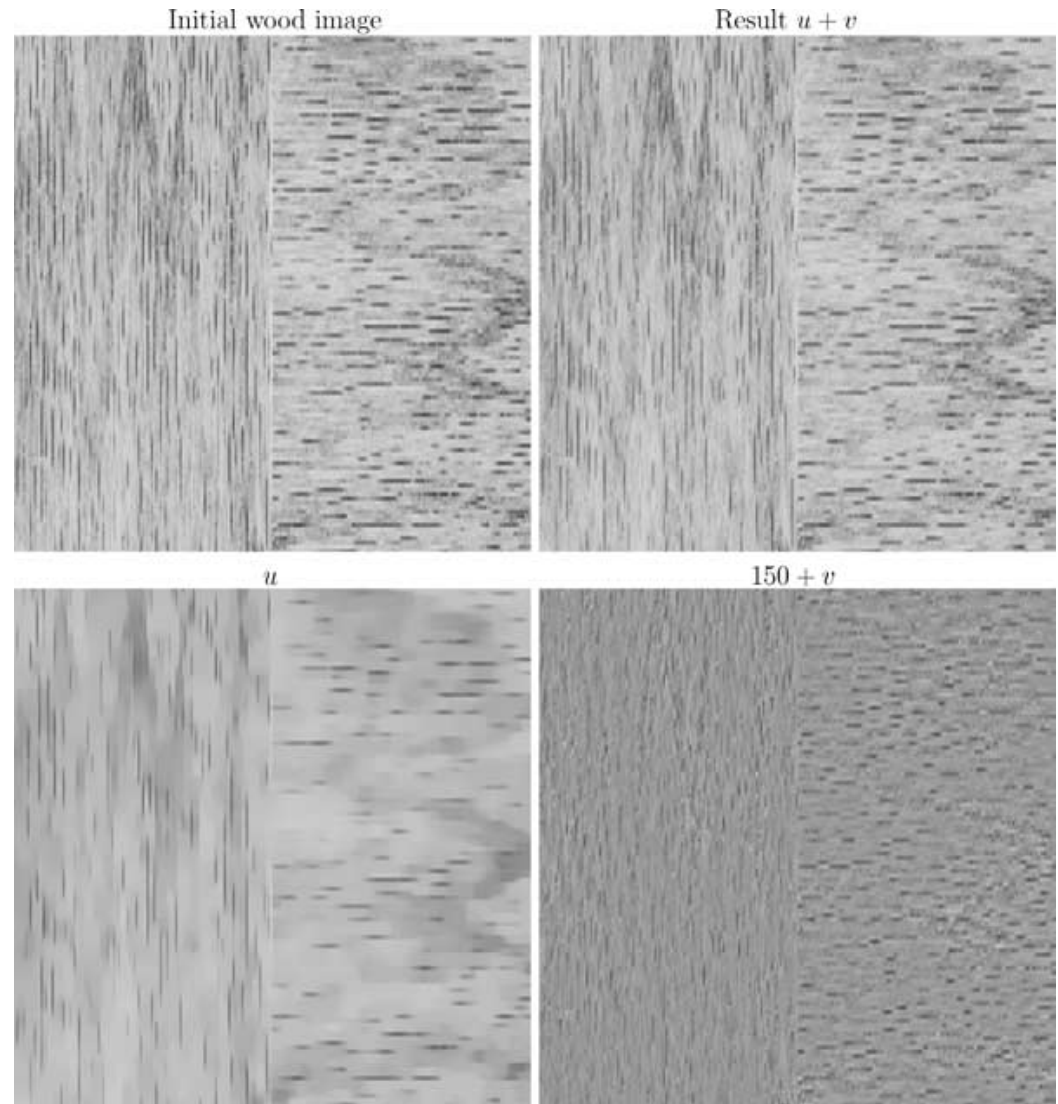

Figure 14. Image decomposition of a wood textured image. 
Moreover, some details of the first chair on the left of the image, which is partially occluded by the desk, are better preserved with the new model. These details are lost in the $u$ component from the standard TV model. So again the new model separates better the cartoon component from the noisy oscillatory components. For each experiment, the parameters are estimated so that the best RMSE is obtained.

We continue the numerical results with an application of the proposed new model to an ultrasound image. In Fig. 7, we show an initial ultrasound image $f$. In Fig. 8, we show the minimizer $u$ obtained with the proposed new model, and the residual $v=f-u$.

We continue the numerical results with an example of image decomposition into cartoon plus texture, obtained by the proposed new model. In Fig. 9 we show an initial textured image $f$. The minimizer $u$ of the proposed new energy is shown in Fig. 10 left, while the residual $f-u$ is shown in Fig. 10 right. We therefore illustrate again how the proposed new model separates and decomposes a given image $f$ into a cartoon part $u$ and an oscillatory part $v=f-u$ of zero mean, such as texture or noise. Again, in this example, we choose $\lambda$ in the new model so that $f-(u+\operatorname{div} \vec{g}) \approx 0$, and the parameter $\mu$ in the new model is very small, to allow as much texture as possible in the $v$ component.

We show in Fig. 11 a comparison between the proposed model from this paper with the fourth order model introduced in [31]. We refer the reader to [31] for more numerical results of image decomposition and image restoration using the fourth order model from [31].

Finally, we end the paper illustrating an important advantage of the proposed models: computing explicitly the functions $g_{1}$ and $g_{2}$, from $v=\operatorname{div} \vec{g}$, we can perform texture discrimination and texture segmentation on some textured images, with two different textures. We show in Figs. 12-15 numerical results already reported in [40]. For two textured images $f$ representing fabric textures and wood textures, we compute the decomposition $u+v$ by the proposed new models. Then, we apply the active contour based segmentation

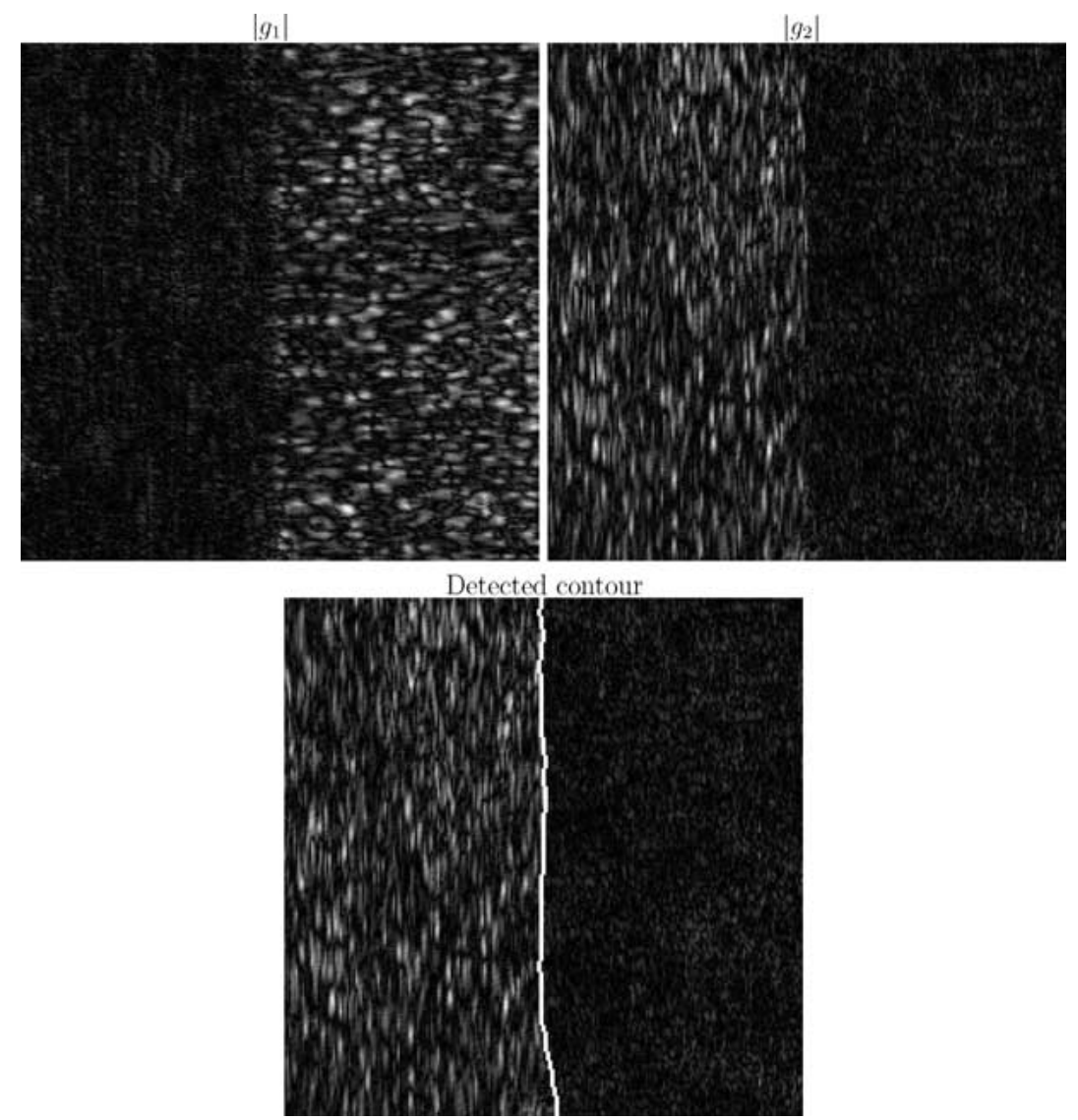

Figure 15. Top: components $\left|g_{1}\right|$ and $\left|g_{2}\right|$ from the wood image. Bottom: detected contour. 
algorithm from $[15,16]$, to one of the components $\left|g_{1}\right|$ or $\left|g_{2}\right|$, to detect the boundary between the textures (at least one of these two components shows different characteristics of the textures). In the wood example, the difference is mainly in the direction of the two textures, while in the fabric texture, there is no preferred direction. However, the textures are well segmented, even in the more difficult case of the fabric texture.

\section{Concluding Remarks}

In this paper, we have presented a new, general and efficient class of models for image denoising and image restoration, which combine the total variation minimization model of Rudin-Osher-Fatemi for image restoration, with dual Sobolev spaces appropriate for oscillatory functions, following ideas introduced by Meyer. Numerical results for image denoising, image decomposition and texture segmentation have been presented. This paper is also an extension of the models introduced by the authors in [40].

\section{Acknowledgments}

The first author would like to thank L. Cohen and the organizers of the Mathematics and Image Analysis Conference, held in Paris, in September 2002, for the invitation to participate to the meeting and to contribute to the special issue. We would also like to mention that the texture image used in the experiment from Figs. 9 and 10 has been obtained from N. Paragios's web page.

\section{References}

1. R. Acar and C.R. Vogel, "Analysis of bounded variation penalty methods of ill-posed problems," Inverse Problem, Vol. 10, pp. 1217-1229, 1994.

2. L. Alvarez, Y. Gousseau, and J.-M. Morel, "Scales in natural images and a consequence on their bounded variation norm," Lecture Notes in Computer Science, Vol. 1682, pp. 247-258, 1999.

3. L. Alvarez, F. Guichard, P.L. Lions, and J.M. Morel, “Axioms and fundamental equations of image-processing," Archive for Rational Mechanics and Analysis, Vol. 123, No. 3, pp. 199-257, 1993.

4. F. Andreu, C. Ballester, V. Caselles, and J.M. Mazon, "Minimizing total variation flow," CRAS I-Mathématique, Vol. 331, No. 11, pp. 867-872, 2000.

5. F. Andreu, V. Caselles, J.I. Diaz, and J.M. Mazon, "Some qualitative properties for the total variation flow," Journal of Functional Analysis, Vol. 188, No. 2, pp. 516-547, 2002.

6. G. Aubert and L. Vese, "A variational method in image recovery," Siam Journal on Numerical Analysis, Vol. 34, No. 5, pp. 1948-1979, 1997.

7. J.-F. Aujol, G. Aubert, L. Blanc-Féraud, and A. Chambolle, "Image decomposition application to SAR images," Lecture Notes in Computer Science, Vol. 2695, pp. 297-312, 2003.

8. C. Ballester and M. Gonzalez, "Affine invariant texture segmentation and shape from texture by variational methods," J. Math. Imaging Vis., Vol. 9, No. 2, pp. 141-171, 1998.

9. M. Bertalmio, L. Vese, G. Sapiro, and S. Osher, "Simultaneous structure texture image inpainting," Transactions on Image Processing, Vol. 12, No. 8, pp. 882-889, 2003.

10. E. J. Candès and F. Guo, "New multiscale transforms, minimum total variation synthesis: Applications to edge-preserving image reconstruction," Signal Processing, Vol. 82, pp. 1519-1543, 2002.

11. S. Casadei, S. Mitter, and P. Perona, "Boundary detection in piecewise homogeneous textured images," Lecture Notes in Computer Science, Vol. 588, pp. 174-183, 1992.

12. A. Chambolle and P.L. Lions, "Image recovery via total variation minimization and related problems," Numer. Math., Vol. 76, pp. 167-188, 1997.

13. A. Chambolle, "An algorithm for total variation minimization and applications," Cahiers de mathématiques du CEREMADE 0240, April 2002, to appear in JMIV, 2003.

14. T.F. Chan, G.H. Golub, and P. Mulet, "A nonlinear primal-dual method for total variation-based image restoration," SIAM J. Scientific Computing, Vol. 20, No. 6, pp. 1964-1977, 1999.

15. T. Chan and L. Vese, "An active contour model without edges," in "Scale-space theories in computer vision," Lecture Notes in Computer Science, Vol. 1682, pp. 141-151, 1999.

16. T.F. Chan and L.A. Vese, "Active contours without edges," IEEE Transactions on Image Processing, Vol. 10, No. 2, pp. 266-277, 2001.

17. I. Daubechies and G. Teschke, "Wavelet based image decomposition by variational functionals," March 2003.

18. L.C. Evans, "Partial differential equations, graduate studies in mathematics," Vol. 19, AMS 1998.

19. Y. Gousseau and J.M. Morel, "Are natural images of bounded variation?" SIAM J. on Mathematical Analysis, Vol. 33, No. 3, pp. 634-648, 2001.

20. R. Kimmel, R. Malladi, and N. Sochen, "Images as embedded maps and minimal surfaces: Movies, color, texture, and volumetric medical images," IJCV, Vol. 39, No. 2, pp. 111-129, 2000.

21. R. Kimmel, N. Sochen, and R. Malladi, The Geometry of Texture, Curves and Surfaces 99, St-Malo, France, March 1999.

22. G. Koepfler, C. Lopez, and J.-M. Morel, “A multiscale algorithm for image segmentation by variational method," SIAM J. Num. Analysis, Vol. 31, No. 1, pp. 282-299, 1994.

23. T.S. Lee, "A Bayesian framework for understanding texture segmentation in the primary visual-cortex," Vision Research, Vol. 35, No. 18, pp. 2643-2657, 1995.

24. T.S. Lee, D. Mumford, and A. Yuille, "Texture segmentation by minimizing vector-valued energy functionals-The coupledmembrane model," Lecture Notes in Computer Science, Vol. 588, pp. 165-173, 1992.

25. F. Malgouyres, "Mathematical analysis of a model which combines total variation and wavelet for image restoration," Technical Report of Université Paris 13.

26. F. Malgouyres, "Minimizing the total variation under a wavelet style constraint for image restoration," UCLA CAM Report 01-23. 
27. Y. Meyer, "Oscillating patterns in image processing and nonlinear evolution equations," University Lecture Series, Vol 22, AMS 2002.

28. D. Mumford and B. Gidas, "Stochastic models for generic images," Quarterly of Applied Mathematics, Vol. 59, No. 1, pp. 85-111, 2001.

29. D. Mumford and J. Shah, "Optimal approximation by piecewise smooth functions and associated variational problems," Comm. Pure Appl. Math., Vol. 42, pp. 577-685, 1989.

30. F. Oru, "Le rôle des oscillations dans quelques problèmes d'analyse non-linéaire," Thèse, CMLA, ENS-Cachan, June 9, 1998.

31. S. Osher, A. Sole, and L. Vese, "Image decomposition and restoration using total variation minimization and the $H^{-1}$ norm," UCLA CAM Report 02-57, October 2002, to appear in SIAM J. of Multiscale Modeling and Simulation.

32. N. Paragios and R. Deriche, "Geodesic active contours and level set methods for supervised texture segmentation," International Journal of Computer Vision, Vol. 46, No. 3, pp. 223-247, 2002.

33. P. Perona and J. Malik, "Scale-space and edge-detection using anisotropic diffusion," IEEE on PAMI, Vol. 12, No. 7, pp. 629639, 1990.

34. L. Rudin and S. Osher, "Total variation based image restoration with free local constraints," in Proc. IEEE ICIP, Vol. I, pp. 3135, Austin, TX, USA, 1994

35. L. Rudin, S. Osher, and E. Fatemi, "Nonlinear total variation based noise removal algorithms," Physica D, Vol. 60, pp. 259268, 1992.

36. C. Sagiv, N. Sochen, and Y.Y. Zeevi, "Generation of the Gaborfeature-space metric for texture segmentation via principal component analysis," IEEE Trans. on Pattern Analysis and Machine Intelligence, Submitted 2002.

37. B. Sandberg, T. Chan, and L. Vese, "A level-set and Gaborbased active contour algorithm for segmenting textured images,' UCLA CAM Report 02-39, 2002.

38. G. Sapiro, "Color snakes," Computer Vision and Image Understanding, Vol. 68, No. 2, pp. 247-253, 1997.

39. J.-L. Starck, M. Elad, and D.L. Donoho, "Image decomposition: Separation of texture from piecewise smooth content," in Proceedings SPIE Conference on Signal and Image Processing, Vol. 5207, M.A. Unser, A. Aldroubi, and A.F. Laine (Eds.), "Wavelets: Applications in signal and image processing X,' SPIE's 48th Annual Meeting, 3-8 August 2003, San Diego, USA.

40. L.A. Vese and S.J. Osher, "Modeling textures with total variation minimization and oscillating patterns in image processing," UCLA CAM Report 02-19, May 2002, to appear in Journal of Scientific Computing, 2003.

41. L. Vese, "A study in the BV space of a denoising-deblurring variational problem," Applied Mathematics and Optimization, Vol. 44, No. 2, pp. 131-161, 2001.

42. S.C. Zhu, Y.N. Wu, and D. Mumford, "Filters, random fields and maximum entropy (FRAME): Towards a unified theory for texture modeling," International Journal of Computer Vision, Vol. 27, No. 2, pp. 107-126, 1998.

43. S.C. Zhu, Y.N. Wu, and D. Mumford, "Minimax entropy principle and its application to texture modeling," Neural Computation, Vol. 9, No. 8, pp. 1627-1660, 1997.

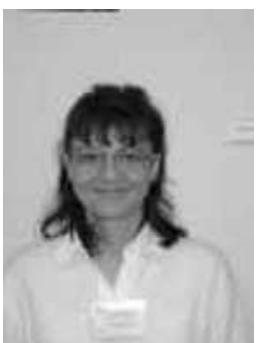

Luminita Vese obtained the B.S. and M.S. in mathematics from the University of Timisoara, Romania (1992), and the M.S. and Ph.D. from the University of Nice Sophia Antipolis, France (1996). Her research interests are in calculus of variations and partial differential equations, with applications to image analysis. She is currently Assistant Professor in the Department of Mathematics, at the University of California, Los Angeles. From 1996 to 2002, she held postdoctoral teaching and research positions at the University of Nice, University of Paris IX-Dauphine, and UCLA.
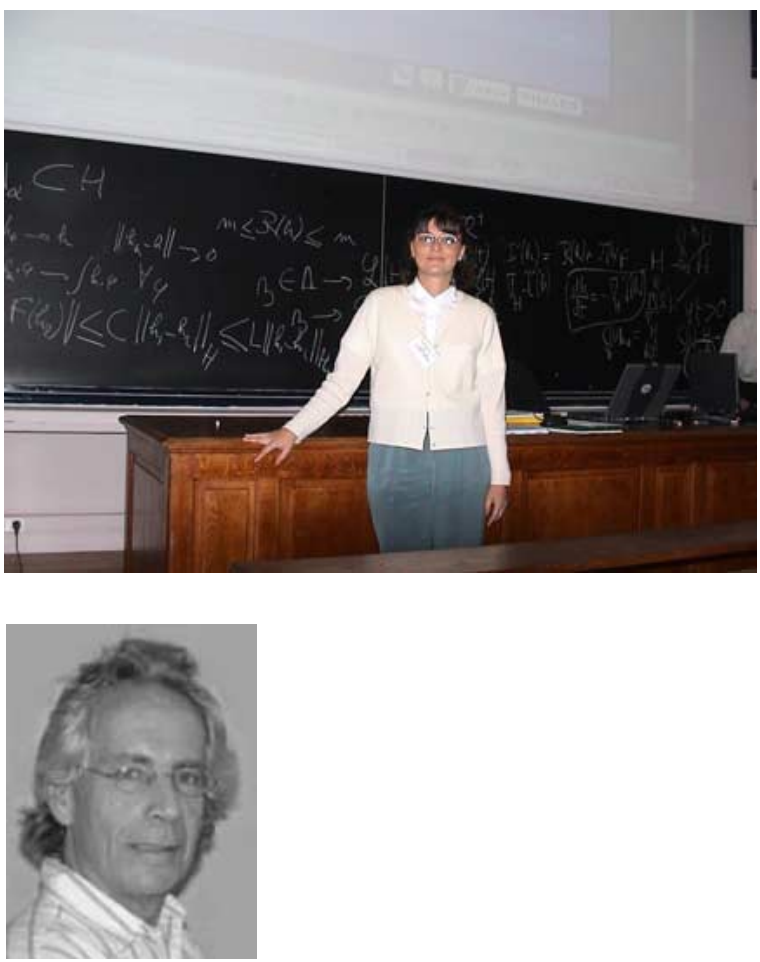

Stanley Osher is Professor of Mathematics at the University of California, Los Angeles (M.S. and Ph.D from NYU, 1964, 1966). His research contributions are in algorithm development and applications in high resolution shock capturing methods, level set methods, and PDE based methods in computer vision and image processing. A few examples are: the Engquist-Osher scheme, TVD schemes, entropy conditions, ENO and WENO schemes, numerical schemes for Hamilton-Jacobi type equations, total variation model for image denoising, images on implicit surfaces, the level set method in image processing and computer vision. He is co-author with R. Fedkiw of the book The Level Set Method and Dynamic Implicit Surfaces. 